\title{
Testicular Spermatocytic Tumor with Sarcoma
}

National Cancer Institute

\section{Source}

National Cancer Institute. Testicular Spermatocytic Tumor with Sarcoma. NCI

Thesaurus. Code C39922.

A malignant germ cell tumor that arises from the testis and is characterized by the presence of a spermatocytic tumor component that is contiguous with an undifferentiated or differentiated sarcomatous component. It usually presents as a slowly growing mass that enlarges very rapidly soon after the initial diagnosis. 